\title{
ELECTRON COOLER IMPEDANCES
}

\author{
A.V. BUROV* \\ Budker INP, 630090, Novosibirsk, Russia
}

(Received 15 July 1996; In final form 2 April 1997)

\begin{abstract}
A cooling electron beam can be considered as a medium which responds to fields generated by a cooled ion beam. Electron density perturbations excited by ion fluctuations give rise to stabilization or destabilization of the ion motion. This reaction on the ion fluctuations can be described in terms of an impedance introduced in the ring by the electron beam. Longitudinal and transverse impedances of the electron cooler are found here, increment (decrement) rates introduced in the ion coherent modes are calculated.
\end{abstract}

Keywords: Storage ring; Ion beam; Electron cooling; Coherent instability

\section{INTRODUCTION}

An electron cooling ${ }^{1,2}$ is proved to be one of efficient methods for a storage of dense ion beams. Hot ions collide with cold electrons of an accompanying electron beam inside a cooling part of the storage ring. In the result, an ion thermal energy is transferred to electrons, and ion phase density increases (details and references concerning electron cooling can be found e.g. in Ref. 3). However, long-range Coulomb forces cause a coherent ion-electron interaction also. This interaction introduces decrements (increments) in various coherent modes of the ion beam, with the rates proportional to the electron current. This problem revealed its importance especially after experiments at an ion storage ring CELSIUS, Uppsala, where a decrease of an ion beam lifetime with an increase of the electron current was

\footnotetext{
*E-mail: Burov@inp.nsk.su.
} 
observed $;{ }^{4}$ the phenomenon is referred to as an 'electron heating'. The intensity-dependent phenomena were also observed at NAP-M, ${ }^{2}$ IUCF $^{5}$ and TARN II. ${ }^{6}$ These effects are not well-understood. It is not clear, in a particular, could the coherent ion-electron interaction be responsible for some of them, as it was suggested in. ${ }^{7}$

Here, an analytical study of the coherent ion-electron interaction is presented. An expression for the electrostatic potential of an arbitrary multipolarity is derived in the next section on a base of the hydrodynamic approximation for the electron medium. Then, longitudinal and transverse impedances introduced by the electron beam are obtained, the coherent stability problems are considered, the conclusions are formulated.

\section{MAIN EQUATIONS}

Density perturbations of a cooled ion beam awake coherent motion in a cooling electron beam. The electrostatic fields induced in the electron medium introduce increments (decrements) in the exciting ion modes. ${ }^{7-9}$ If the electron beam is good enough, it can be considered as a cold plasma, with the Debye radius $r_{\mathrm{D}}$ much less than the electron and ion beam radii $a, a_{\mathrm{i}}$. In this case, the electron temperature does not influence the coherent ion-electron interaction, their thermal motion can be neglected and a hydrodynamic model ${ }^{10}$ be applied.

In a reference frame, the dynamics of the magnetized electron medium excited by fluctuations of the cooled ion beam can be described by the following set of equations:

$$
\begin{aligned}
\frac{\partial \tilde{n}_{\mathrm{e}}}{\partial t}+\bar{n}_{\mathrm{e}} \frac{\partial \tilde{v}_{\mathrm{e}}}{\partial z} & =0 \\
\frac{\partial \tilde{v}_{\mathrm{e}}}{\partial t}-\frac{e}{m} \frac{\partial \Phi}{\partial z} & =0 \\
\frac{1}{r} \frac{\partial}{\partial r}\left(r \frac{\partial \Phi}{\partial r}\right)+\frac{\partial^{2} \Phi}{\partial z^{2}}+\frac{1}{r^{2}} \frac{\partial^{2} \Phi}{\partial \phi^{2}} & =4 \pi e\left(\tilde{n}_{\mathrm{e}}-\tilde{n}_{\mathrm{i}}\right) .
\end{aligned}
$$

Here $\bar{n}_{\mathrm{e}}$ is a bulk electron density, $\tilde{n}_{\mathrm{e}}, \tilde{n}_{\mathrm{i}}$ are electron and ion density perturbations, $\tilde{v}_{\mathrm{e}}$ is a perturbation of electron velocity, $\Phi$ is an 
electrostatic potential. A magnetic field implied here to be directed along the longitudinal axis $z$, which neglects an influence of a bended entrance part of the cooler. The electron medium integrates the influence of ion perturbations for the plasma time $\simeq \omega_{\mathrm{e}}^{-1}, \omega_{\mathrm{e}}=$ $\sqrt{4 \pi \bar{n}_{\mathrm{e}} e^{2} / m}$; a contribution of the bended part can be neglected if a phase advance $\omega_{\mathrm{e}} \tau_{\text {bend }} \ll 1$, which is normally satisfied.

The equations of motion (1) have to be supplemented by zero initial conditions imposed on the electron perturbations at the ion entrance in the electron beam:

$$
\left.\tilde{n}_{\mathrm{e}}\right|_{z=-v t}=0,\left.\quad \tilde{v}_{\mathrm{e}}\right|_{z=-v t}=0 .
$$

The solution of the problem with the initial conditions (Eq. (2)) can be presented as a sum of a particular solution of Eqs. (1) and a general solution of the homogeneous set, with $\tilde{n}_{\mathrm{i}}=0$. The arbitrary constants of the general solution are found from the boundary conditions.

Looking for the general solution as $\propto \exp (i q z-i \omega t) \cos (l \phi)$, the result writes

$$
\begin{gathered}
\Phi(r)= \begin{cases}A J_{l}(\kappa r), & r \leq a, \\
B K_{l}(q r)+C I_{l}(q r), & r \geq a,\end{cases} \\
\tilde{v}_{\mathrm{e}}=-\frac{e}{m} \frac{q}{\omega} \Phi, \quad \tilde{n}_{\mathrm{e}}=-\frac{\bar{n}_{\mathrm{e}} e}{m} \frac{q^{2}}{\omega^{2}} \Phi, \quad \omega=\omega_{ \pm}= \pm q \omega_{\mathrm{e}} / \sqrt{q^{2}+\kappa^{2},}
\end{gathered}
$$

where $J_{l}$ is the Bessel function, $K_{l}, I_{l}$ are modified Bessel functions. The constants $B, C$ are specified by the constant $A$ : due to electric field continuity,

$$
\left.\Phi\right|_{r=a-0}=\left.\Phi\right|_{r=a+0},\left.\quad \frac{\partial \Phi}{\partial r}\right|_{r=a-0}=\left.\frac{\partial \Phi}{\partial r}\right|_{r=a+0} .
$$

The requirement for the potential to be zero on the vacuum chamber surface

$$
\Phi(b)=0
$$

gives a sequence of eigennumbers $\kappa=\kappa_{l \mu}(q)$ with $\mu=1,2,3, \ldots$ as a radial mode counter. 
The eigenfrequencies are described by the dispersion equation (3):

$$
\omega_{l \mu \pm}(q)= \pm \omega_{l \mu}(q)= \pm q u_{l \mu}(q), \quad u_{l \mu}(q)=\omega_{\mathrm{e}} / \sqrt{q^{2}+\kappa_{l \mu}^{2}} .
$$

The solution of the problem is simplified for two opposite cases. The first corresponds to the vacuum chamber to be radially remote from the electron beam: $b \gg a, q^{-1}$; the second - almost to touch it: $b-a \ll b$. In the first case, when the vacuum chamber is wide, the eigennumbers $\kappa_{l \mu}$ are found from $C=0$. This condition together with Eqs. (4) is satisfied when the transverse wave number $\kappa$ is a zero of the Bessel function $J_{l-1}$ :

$$
\begin{aligned}
& J_{l-1}\left(\kappa_{l \mu} a\right)=0, \quad(l, \mu) \neq(0,1), \\
& \kappa_{01}=a^{-1} \sqrt{2 /(\ln (1 /(q a))+1 / 2)}, \quad 1 / b \ll q<1 / a .
\end{aligned}
$$

If the metal surface of the vacuum chamber adjoins the electron beam, the eigennumbers are zeros of the Bessel function $J_{l}$ :

$$
J_{l}\left(\kappa_{l \mu}\right)=0, \quad b-a \ll a .
$$

In a general case, the transverse wave numbers $\kappa_{l \mu}=\kappa_{l \mu}(q)$ have to found from the following equation:

$$
\begin{aligned}
& K_{l}(q b)\left[q I_{l}^{\prime}(q a) J_{l}\left(\kappa_{l \mu} a\right)-\kappa_{l \mu} I_{l}(q a) J_{l}^{\prime}\left(\kappa_{l \mu} a\right)\right] \\
& \quad-I_{l}(q b)\left[q K_{l}^{\prime}(q a) J_{l}\left(\kappa_{l \mu} a\right)-\kappa_{l \mu} K_{l}(q a) J_{l}^{\prime}\left(\kappa_{l \mu} a\right)\right]=0,
\end{aligned}
$$

gives for a given longitudinal wave number $q$; the primes denote derivatives over arguments. The solutions of the homogeneous problem form a complete orthogonal set, the orthogonality relations

$$
\int_{0}^{a} J_{l}\left(\kappa_{l \mu} r\right) J_{l}\left(\kappa_{l \nu} r\right) r \mathrm{~d} r=\delta_{\mu \nu} \frac{a^{2}}{2} F_{l \mu}^{2}
$$

are satisfied; in the limit cases

$$
F_{l \mu}= \begin{cases}J_{l}\left(\kappa_{l \mu} a\right) & \text { if } q b \gg 1, \\ J_{l}^{\prime}\left(\kappa_{l \mu} a\right) & \text { if } b-a \ll b .\end{cases}
$$


Thus, the basic set of solutions for the homogeneous problem (Eqs. (1) with $\tilde{n}_{\mathrm{i}}=0$ ) is found; at $r \leq a$

$$
\begin{aligned}
& \Phi=A_{l \mu \pm} J_{l}\left(\kappa_{l \mu} r\right) \cos (l \phi) \exp \left(i q z \mp i \omega_{l \mu} t\right), \\
& \tilde{v}_{\mathrm{e}}=- \pm \frac{e}{m} \frac{A_{l \mu \pm}}{u_{l \mu}} J_{l}\left(\kappa_{l \mu} r\right) \cos (l \phi) \exp \left(i q z \mp i \omega_{l \mu} t\right), \\
& \tilde{n}_{\mathrm{e}}=-\frac{\omega_{\mathrm{e}}^{2}}{4 \pi e} \frac{A_{l \mu \pm}}{u_{l \mu}^{2}} J_{l}\left(\kappa_{l \mu} r\right) \cos (l \phi) \exp \left(i q z \mp i \omega_{l \mu} t\right) .
\end{aligned}
$$

Turning to the particular solution, let us present the ion density perturbation as expanded over this basis:

$$
\begin{gathered}
\tilde{n}_{\mathrm{i}}=\tilde{n}_{\mathrm{i} l \mu} J_{l}\left(\kappa_{l \mu} r\right) \cos (l \phi) \exp \left(i k z-i \omega_{\mathrm{i}} t\right), \quad \omega_{\mathrm{i}}=l \omega_{\mathrm{b}} t+k \delta v t, \\
\tilde{n}_{\mathrm{i} l \mu}=\frac{2}{a^{2} F_{l \mu}^{2}} \int_{0}^{a} \tilde{n}_{\mathrm{il} l}(r) J_{l}\left(\kappa_{l \mu} r\right) r \mathrm{~d} r, \\
\tilde{n}_{\mathrm{i} l}(r)=\frac{1}{\pi\left(1+\delta_{l 0}\right)} \int_{0}^{2 \pi} \tilde{n}_{\mathrm{i}}(r, \phi) \cos (l \phi) \mathrm{d} \phi,
\end{gathered}
$$

where $\omega_{\mathrm{b}}$ is a betatron frequency and $\delta v=v_{\mathrm{i}}-v_{\mathrm{e}} \ll v$ is an ion-electron velocity detuning. For this driving term, the particular solution has the same space-time structure:

$$
\Phi=\left(1-g_{\mu}\right) \frac{4 \pi e \tilde{n}_{\mathrm{i}}}{k^{2}+\kappa^{2}}, \quad \tilde{n}_{\mathrm{e}}=g_{\mu} \tilde{n}_{\mathrm{i}}, \quad \tilde{v}_{\mathrm{e}}=g_{\mu} \frac{\omega_{\mathrm{i}}}{k} \frac{\tilde{n}_{\mathrm{i}}}{\bar{n}_{\mathrm{e}}}
$$

where

$$
g_{\mu}=\frac{\omega_{\mu}^{2}(k)}{\omega_{\mu}^{2}(k)-\omega_{\mathrm{i}}^{2}}
$$

is a screening factor describing the reaction of the electron medium on external perturbations at the frequency $\omega_{\mathrm{i}}$.

The solution of the problem (1) consists of the particular solution (13) and a certain solution of the homogeneous problem, such that the initial conditions (2) are satisfied.

A time dependence of the electron perturbation $\propto \exp (i q z-i \omega t)$ at the ion entrance $z=-v t$ has to be the same as a time dependence of 
the ion exciting perturbation $\propto \exp \left(-i k v t-i \omega_{\mathrm{i}} t\right)=\exp \left(-i q v t \mp i \omega_{l \mu} t\right)$, hence,

$$
q=q_{l \mu \pm}=k+\omega_{\mathrm{i}} / v \mp \omega_{l \mu}(q) / v .
$$

This requirement determines the (Doppler) connection between the electron and ion longitudinal wave numbers. Normally, the electron beam is rather diluted,

$$
\alpha \equiv \omega / k v=u / v \ll 1,
$$

the $( \pm)$ wave numbers are almost equal: $q_{-} \approx q_{+}, \omega_{-} \approx-\omega_{+}$,

$$
\left(q_{-}-q_{+}\right) / k=2 \alpha ; \quad\left(\omega_{-}+\omega_{+}\right) /(k u)=-2 \alpha \kappa^{2} /\left(\kappa^{2}+q^{2}\right),
$$

the subscripts $l, \mu$ are implied. Retention of the small terms $\propto \alpha$ is caused by a mutual cancellation of leading $( \pm)$ terms in a total electron response (see below). The wave amplitudes corresponding to the zero initial conditions (2) follows:

$$
\frac{A_{\mu \pm}}{u_{\mu \pm}}=2 \pi e \tilde{n}_{\mathrm{i} \mu} \frac{u_{\mu}(k)}{\omega_{\mathrm{e}}^{2}} \frac{\omega_{\mu}(k)}{\omega_{\mu}(k) \mp \omega_{\mathrm{i}}} .
$$

When the wave amplitudes are found, the particular solution (13) can be forgotten. This part of the total solution describes an instant reaction of the electron medium on the external perturbation, it does not cause energy losses and instabilities and is responsible for small frequency shifts only. On the contrary, the wave part of the total electron reaction (11) is concerned with the energy losses and can give rise to the instabilities of the ion beam.

The action of the electron waves on the ions is time-dependent and have to be integrated (averaged) over time of a pass through the cooler $\tau$. Taking into account the small velocity detuning $\delta v$ and the ion betatron motion, this averaging reduces to a change of timedependent exponents in (11) on a time-transit factor:

$$
\begin{aligned}
& \left\langle\exp \left(i q \delta v t-i \omega t+i l \omega_{\mathrm{b}} t\right)\right\rangle \\
& \quad=\frac{1}{\tau} \int_{0}^{\tau} \exp \left(-i \omega t+i \omega_{\mathrm{i}} t\right) \mathrm{d} t \rightarrow-i T(\Delta \psi)
\end{aligned}
$$




$$
T(\Delta \psi)=\frac{1-\cos \Delta \psi}{\Delta \psi}, \quad \Delta \psi=\left(\omega-\omega_{\mathrm{i}}\right) \tau
$$

Here an insignificant real part of the integral is omitted.

Summing up, the averaged wave potential writes

$$
\begin{aligned}
&\langle\Phi\rangle=-i \frac{2 \pi e}{\omega_{\mathrm{e}}^{2}} \cos (l \phi) \sum_{\mu} J_{l}\left(\kappa_{l \mu} r\right) u_{\mu}(k) \tilde{n}_{\mathrm{i} l \mu} \\
& \times\left[\frac{u_{\mu+} \omega_{\mu}(k)}{\omega_{\mu}(k)-\omega_{\mathrm{i}}} T\left(\Delta \psi_{\mu+}\right) \exp \left(i q_{\mu+} z\right)\right. \\
&\left.+\frac{u_{\mu-} \omega_{\mu}(k)}{\omega_{\mu}(k)+\omega_{\mathrm{i}}} T\left(\Delta \psi_{\mu-}\right) \exp \left(i q_{\mu-} z\right)\right], \\
& \Delta \psi_{\mu \pm}=\left(\omega_{\mu \pm}-\omega_{\mathrm{i}}\right) \tau ; \quad \omega_{\mu-} \approx-\omega_{\mu+} .
\end{aligned}
$$

In the following calculations of the spectral response functions (impedances), the longitudinal coordinate $z \rightarrow 0$, and the transverse radial dependence is finally averaged over the ion beam radial distribution. The leading terms in the result obtained do not contain the small factors $\propto \alpha=\left(q_{-}-q_{+}\right) / 2 k,(16)$ and $\propto \psi_{\mathrm{i}}=\omega_{\mathrm{i}} \tau$. However, these leading contributions from $(+)$ and $(-)$ waves cancel each other: a decelerating action of the positive wave is almost compensated by an accelerating action of the negative one. The real parts of impedances are calculated as a first-order terms in an expansion of the general result over small parameters $\alpha$ and $\Delta \psi_{+}^{2}-\Delta \psi_{-}^{2}$.

\section{LONGITUDINAL IMPEDANCE}

In this chapter longitudinal perturbations are calculated: $l=0$, $\tilde{n}_{\mathrm{i}}(r)=\bar{n}_{\mathrm{i}}(r) \tilde{\rho}_{\mathrm{i}} / \bar{\rho}_{\mathrm{i}}$,

$$
\tilde{n}_{\mathrm{i} \mu}=\frac{\bar{J}_{0 \mu} \tilde{\rho}}{\pi a^{2} F_{0 \mu}^{2}}, \quad \bar{J}_{0 \mu}=\frac{\int_{0}^{a} J_{0}\left(\kappa_{\mu} r / a\right) \bar{n}_{\mathrm{i}}(r) r \mathrm{~d} r}{\int_{0}^{\infty} \bar{n}_{\mathrm{i}}(r) r \mathrm{~d} r},
$$

where $\rho_{\mathrm{i}}=2 \pi \int n_{\mathrm{i}}(r) r \mathrm{~d} r$ is ion linear density, the tilde and bar corresponds to the perturbation and unperturbed values. The response of 
the electron medium on the ion perturbation $\tilde{\rho}_{\mathrm{i} 0}$ can be described in terms of a longitudinal impedance $Z^{\| 1}:^{11}$

$$
\left\langle\widehat{E}_{z}\right\rangle \tau=-\tau \frac{\mathrm{d}\langle\hat{\Phi}\rangle}{\mathrm{d} z}=-e \tilde{\rho}_{\mathrm{i} 0} Z^{\|},
$$

a hat denotes the averaging over radial ion distribution: $\hat{\Phi}=$ $\int \Phi(r) \bar{n}_{\mathrm{i}}(r) r \mathrm{~d} r / \int \bar{n}_{\mathrm{i}}(r) r \mathrm{~d} r$.

Making use of the formulae above (Eqs. (20), (21)), the longitudinal impedance writes:

$$
\begin{aligned}
\operatorname{Re} Z^{\|}= & \frac{2}{\omega_{\mathrm{e}}^{2} a^{2}} \sum_{\mu} \mathcal{K}_{\mu} u_{\mu}\left[\frac{\psi_{\mu} \psi_{\mu+}}{\psi_{\mu}-\psi_{\mathrm{i}}} \frac{1-\cos \left(\Delta \psi_{\mu+}\right)}{\Delta \psi_{\mu+}}\right. \\
& \left.-\frac{\psi_{\mu} \psi_{\mu-}}{\psi_{\mu}+\psi_{\mathrm{i}}} \frac{1-\cos \left(\Delta \psi_{\mu-}\right)}{\Delta \psi_{\mu-}}\right], \\
\mathcal{K}_{\mu}= & \bar{J}_{\mu}^{2} / F_{\mu}^{2}, \quad \psi_{\mu}=\omega_{\mu}(k) \tau, \quad \psi_{\mu \pm}=\omega_{\mu \pm} \tau \\
\psi_{\mathrm{i}}= & k \delta v \tau, \quad \Delta \psi_{\mu \pm}=\psi_{\mu \pm}-\psi_{\mathrm{i}} .
\end{aligned}
$$

First, let us calculate (23) without the velocity detuning. This part of the impedance is found by a substitution $\psi_{\mathrm{i}}=0$ and expansion of the expression over the small parameter $\alpha$. Taking into account relativistic compression of longitudinal lengths in the laboratory frame, substituting $q \rightarrow k / \gamma$, assuming $k$ as a laboratory frame wave number, the result follows:

$$
\begin{aligned}
& \operatorname{Re} Z^{\|}(q)=-\frac{Z_{0} \psi_{\mathrm{e}}}{\pi \beta} S_{0}^{\|}(q), \quad S_{0}^{\|}(q)=\sum_{\mu} \frac{\mathcal{K}_{\mu} \kappa_{\mu}^{2} q \sin \psi_{\mu}}{a^{2}\left(\kappa_{\mu}^{2}+q^{2}\right)^{5 / 2}} \\
& q=k / \gamma, \quad \delta v=0, \\
& \psi_{\mu}=\omega_{\mu} \tau=\psi_{\mathrm{e}} q / \sqrt{q^{2}+\kappa^{2}}, \quad \psi_{\mathrm{e}}=\omega_{\mathrm{e}} \tau,
\end{aligned}
$$

where $Z_{0}=4 \pi / c=377 \Omega, \beta=v / c$. All the phase advances $\psi_{\mathrm{e}}, \psi_{\mu}$ are relativistic invariants and can be taken in an arbitrary frame. A plot of the mode sum $S_{0}^{\|}(q)$ for certain parameters of the cooler $\left(\psi_{\mathrm{e}}=3.5, b-a \gg a\right)$ is presented on Figure 1. A maximum of this function is reached at $q \simeq 1 / a$ and depends on the formfactors $\bar{J}_{0 \mu}^{2}$. 


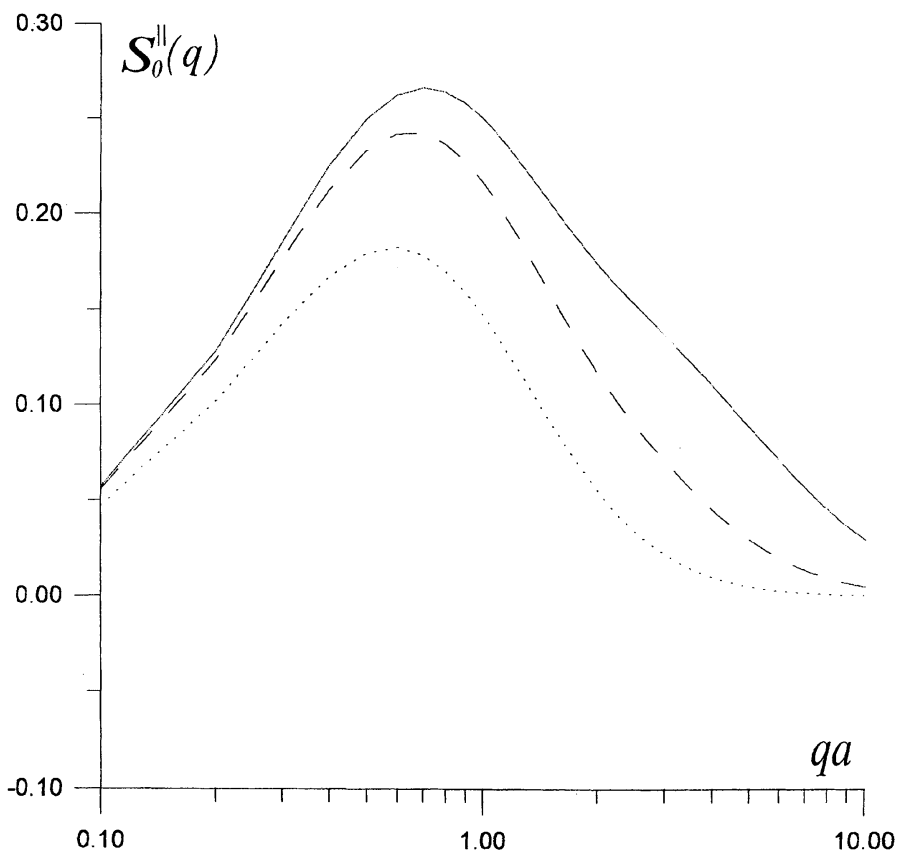

FIGURE 1 The mode sum factor $S_{0}^{\|}(q)$ determines the longitudinal impedance for equal velocities of the electron and ion beams. The ion transverse distribution assumed to be Gaussian with the r.m.s. radius $a_{\mathrm{i}}$, the electron distribution is taken as a step with the radius $a$. Cases $a_{\mathrm{i}} / a=0.1,0.2,0.4$ are shown by the solid, dash and dot lines. The phase advance of the Langmuir plasma oscillations $\omega_{\mathrm{e}} \tau=2.7$, the vacuum chamber is wide: $b-a \gg a$.

If the ion beam radius is small enough, $a_{\mathrm{i}} \ll a$, then $\bar{J}_{01}^{2} \approx 1$; when the phase advance $\psi_{\mathrm{e}}>1$, it gives $\max _{q}\left|S_{0}^{\|}(q)\right| \approx 0.3$ and $^{10}$

$$
\max _{k} \operatorname{Re} Z^{\|}(k) \approx \operatorname{Re} Z^{\|}(\gamma / a) \approx 0.1 Z_{0} \psi_{\mathrm{e}} / \beta .
$$

To calculate the detuning-dependent part of the impedance, the small terms $\propto \alpha$ can be neglected, which gives

$$
\begin{aligned}
\operatorname{Re} Z^{\|}= & \frac{Z_{0} \psi_{\mathrm{e}}^{2}}{\pi \beta} \frac{\ell}{\gamma a} \sum_{\mu} \frac{\mathcal{K}_{\mu} q^{3}}{a\left(\kappa_{\mu}^{2}+q^{2}\right)^{2}} \frac{1}{2 \psi_{\mu}} \\
& \times\left[\frac{1-\cos \left(\psi_{\mathrm{i}}-\psi_{\mu}\right)}{\left(\psi_{\mathrm{i}}-\psi_{\mu}\right)^{2}}-\frac{1-\cos \left(\psi_{\mathrm{i}}+\psi_{\mu}\right)}{\left(\psi_{\mathrm{i}}+\psi_{\mu}\right)^{2}}\right],
\end{aligned}
$$


where $\ell$ is the cooler length in the laboratory frame. When the phase advances $\psi_{\mathrm{i}}, \psi_{\mu}$ satisfy some limit conditions, this general expression can be simplified:

$$
\begin{aligned}
& \operatorname{Re} Z^{\|}(k)= \frac{Z_{0} \psi_{\mathrm{e}}^{2}}{\pi \beta} \frac{\ell}{\gamma a} \frac{\mathrm{d}}{\mathrm{d} \psi_{\mathrm{i}}}\left(\frac{\cos \psi_{\mathrm{i}}-1}{\psi_{\mathrm{i}}^{2}}\right) \\
& \times \sum_{\mu} \frac{\mathcal{K}_{\mu} q^{3}}{a\left(\kappa_{\mu}^{2}+q^{2}\right)^{2}} \frac{\sin \psi_{\mu}}{\psi_{\mu}}, \quad \psi_{\mathrm{i}} \gg \psi_{\mu}, \\
& \operatorname{Re} Z^{\|}(k)= \frac{Z_{0} \psi_{\mathrm{e}}^{2}}{\pi \beta} \frac{\ell}{\gamma a} \sin \psi_{\mathrm{i}} \sum_{\mu} \frac{\mathcal{K}_{\mu} q^{3}}{a\left(\kappa_{\mu}^{2}+q^{2}\right)^{2}} \\
& \times \frac{1}{\psi_{\mu}} \frac{\mathrm{d}}{\mathrm{d} \psi_{\mu}}\left(\frac{\cos \psi_{\mu}-1}{\psi_{\mu}^{2}}\right), \quad \psi_{\mu} \gg \psi_{\mathrm{i}}, \\
& q=k / \gamma, \quad \psi_{\mathrm{i}}=q \delta v \tau=\frac{k a}{\gamma} \frac{\delta p}{p} \frac{\ell}{\gamma a},
\end{aligned}
$$

where $\delta p / p$ is a relative momentum detuning in the laboratory frame.

\section{LONGITUDINAL STABILITY}

The calculated impedances depend on the velocity detuning $\propto \delta p / p$. However, there are two kinds of the detuning. The first is a systematic, time-independent discrepancy. Beam stability problems can be treated with it in a standard way. The second kind of the detuning origins in synchrotron oscillations of ions. Due to this kind of relative motion, the calculated impedances prove to be time-dependent; therefore, the stability problems need a particular analysis. To calculate a coherent spectrum in this case, the impedance dependence on the momentum $\delta p / p$ have to be taken into account at the averaging of the perturbative coherent interaction over synchrotron oscillations. Generally, the complex coherent frequency shift $\Delta \Omega_{m}$ of the synchrotron mode $m$ is determined by a certain functional of the impedance,

$$
\begin{gathered}
\Delta \Omega_{m} \propto i \int_{-\infty}^{\infty} \mathrm{d} k F(k) \int_{0}^{2 \pi} \mathrm{d} \theta Z^{\|}(k, \delta p / p) \delta p / p \exp (i k z-i m \theta), \\
\delta p / p=\delta p_{0} / p \sin \theta, \quad z=z_{0} \cos \theta
\end{gathered}
$$


with some function $F(k)$. The considered interaction is local: the impedance reaches a maximum at high wave numbers, $k z_{0} \simeq l_{\mathrm{b}} / a \gg 1$, where $l_{\mathrm{b}}$ is the bunch length. In this case, the saddle-point method can be used for the calculation of the integral over synchrotron phase $\theta$; the main contribution in the integral is concentrated in a small interval $\Delta \theta=\left(\mathrm{d}^{2} / \mathrm{d} \theta^{2}(k z-m \theta)\right)^{-1 / 2}$ in a vicinity of the saddle-point $\theta=\theta_{0}$, where $\mathrm{d} / \mathrm{d} \theta(k z-m \theta)=0$ :

$$
\sin \theta_{0}=-\frac{m}{k z_{0}}, \quad \Delta \theta=\left(k^{2} z_{0}^{2}-m^{2}\right)^{-1 / 4} \ll 1 .
$$

The factor $Z^{\|}(k, \delta p / p) \delta p / p$ varies with the phase $\theta$ much slower than the exponent; therefore, the synchrotron phase can be changed by its saddle-point value (30):

$$
\begin{gathered}
\frac{\delta p}{p}=\frac{\delta p_{0}}{p} \sin \theta \rightarrow-\frac{\delta p_{0}}{p} \frac{m}{k z_{0}}=-2 \pi m \frac{\nu_{\mathrm{s}}}{\eta k \Pi}, \\
\psi_{\mathrm{i}}=q \delta v \tau=\frac{\delta p}{p} \frac{k \ell}{\gamma^{2}} \rightarrow-\frac{2 \pi m}{m_{\mathrm{i}}}, \quad m_{\mathrm{i}}^{-1}=\frac{\nu_{\mathrm{s}}}{\gamma^{2} \eta} \frac{\ell}{\Pi},
\end{gathered}
$$

where $\nu_{\mathrm{s}}=\Omega_{\mathrm{s}} / \omega_{0}$ is the synchrotron tune, $\Pi$ is the ring circumference, $\eta=\mathrm{d}\left(\ln \omega_{0}\right) / \mathrm{d}(\ln p)$. Thus, the detuning phase advance $\psi_{\mathrm{i}}$ caused by the synchrotron oscillations can be substituted in the impedance dependencies (26), (28) by a certain constant (31) proportional to the mode number $m$; after that, the conventional formulae of the coherent spectrum (see e.g. Ref. 12) can be used.

Assuming a longitudinal ion distribution to be air-bag, $f(\delta p, z) \propto \delta\left(\left(\delta p / \delta p_{\mathrm{b}}\right)^{2}+\left(z / l_{\mathrm{b}}\right)^{2}-1\right)$, the increment rate $\Lambda_{m}=\operatorname{Im} \Delta \Omega_{m}$ writes $^{12}$

$$
\begin{aligned}
\Lambda_{m} & =\frac{4 N r_{\mathrm{i}} c \eta}{\pi \gamma \nu_{\mathrm{s}} l_{\mathrm{b}}^{2}} m \int_{0}^{\infty} \frac{\mathrm{d} k}{k} \frac{\operatorname{Re} Z^{\|}(k)}{Z_{0}} J_{m}^{2}\left(k l_{\mathrm{b}}\right) \\
& \approx \frac{4 N r_{\mathrm{i}} c \eta}{\pi^{2} \gamma^{2} \nu_{\mathrm{s}} l_{\mathrm{b}}^{3}} m \int_{q_{m}}^{\infty} \frac{\mathrm{d} q}{q^{2}} \frac{\operatorname{Re} Z^{\|}}{Z_{0}}, \quad q_{m}=\frac{m}{\gamma l_{\mathrm{b}}},
\end{aligned}
$$

where $r_{\mathrm{i}}=Z_{\mathrm{i}}^{2} e^{2} /\left(M_{\mathrm{i}} c^{2}\right)$ is ion classical radius, $N$ is a number of ions per bunch with the length $l_{\mathrm{b}}$; an asymptotic expression for the Bessel function was used and averaged over fast oscillations. Substituting the impedance (27), neglecting the weak dependence of the transverse 
eigennumbers $\kappa$ on the longitudinal wave number $q$, assuming $\sin \psi_{\mu}$ / $\psi_{\mu} \simeq 1$, the integral (32) is taken:

$$
\begin{gathered}
\frac{\Lambda_{m}}{m}=-\frac{2 N r_{\mathrm{i}} c \eta \psi_{\mathrm{e}}^{2} \ell}{\pi^{3} \gamma^{3} \beta \nu_{\mathrm{s}} l_{\mathrm{b}}^{3}} h^{\prime}(\psi) G^{\|}(Q) ; \quad m_{\mathrm{b}}=\gamma l_{\mathrm{b}} / a, \quad \psi=2 \pi \frac{m}{m_{\mathrm{i}}}, \quad Q=\frac{m}{m_{\mathrm{b}}}, \\
h=\frac{\cos \psi-1}{\psi^{2}}, \quad h^{\prime}(\psi)=\frac{\mathrm{d} h}{\mathrm{~d} \psi}, \quad G^{\|}(Q)=\sum_{\mu} \frac{\mathcal{K}_{\mu}}{\kappa_{\mu}^{2} a^{2}+Q^{2}} .
\end{gathered}
$$

Plots of the functions $h^{\|}(\psi), G^{\|}(Q)$ are shown on Figures 2 and 3. The increment (33) is negative when $m<m_{\mathrm{i}}$, then it changes the sign, which corresponds to an instability. The maximum of the growth rate $\Lambda_{m}$ (33) is determined by the function $h^{\prime}(\psi)$, other factors are significantly slower; it is reached at $m=m_{*} \approx 1.2 m_{\mathrm{i}}$, where $h^{\prime} \approx-1.4 \cdot 10^{-2}$. From here, the maximum growth rate writes

$$
\begin{gathered}
\max _{m} \frac{\Lambda_{m}}{m \Omega_{\mathrm{s}}} \approx \frac{\Lambda_{\max }}{m_{*} \Omega_{\mathrm{s}}}=0.9 \cdot 10^{-3} \frac{N r_{\mathrm{i}} \eta \psi_{\mathrm{e}}^{2} \ell \Pi}{\gamma^{3} \beta^{2} \nu_{\mathrm{s}}^{2} l_{\mathrm{b}}^{3}} G\left(Q_{*}\right) ; \\
Q_{*}=\frac{m_{*}}{m_{\mathrm{b}}}=1.2 \frac{\gamma \eta a \Pi}{\nu_{\mathrm{s}} \ell l_{\mathrm{b}}} .
\end{gathered}
$$

The ion beam motion can be stabilized by the Landau damping, which is not taken into account by the air-bag model. The specific conditions of the damping depend on is it fast or slow in comparison with the synchrotron motion. ${ }^{8}$ For a given synchrotron frequency dispersion $\Delta \Omega_{\mathrm{s}}$, the motion can be considered as slow up to the mode number $m=\Omega_{\mathrm{s}} / \Delta \Omega_{\mathrm{s}}$. At higher numbers, the beam can be considered as a coasting one. For the interested area $m \geq m_{\mathrm{i}}$, the last case is practically typical. It means that the considered microwave motion is stabilized when the Keil-Schnell condition is satisfied:

$$
\left(\frac{\Delta p}{p}\right)^{2} \geq \frac{\bar{\rho}_{\mathrm{i}} r_{\mathrm{i}} c}{2 \pi|\eta| \gamma \beta}\left|\frac{Z_{\mathrm{tot}}^{\|}}{n}\right|, \quad n=k \bar{R}=k \Pi /(2 \pi) .
$$

Here $\bar{\rho}_{\mathrm{i}}=N /\left(\sqrt{2 \pi} l_{\mathrm{b}}\right)$ is a pike ion density, $Z_{\mathrm{tot}}^{\|}$is a total impedance of the ring, typically determined by a dominated contribution of the space charge $Z_{\mathrm{sc}}^{\|}$: 


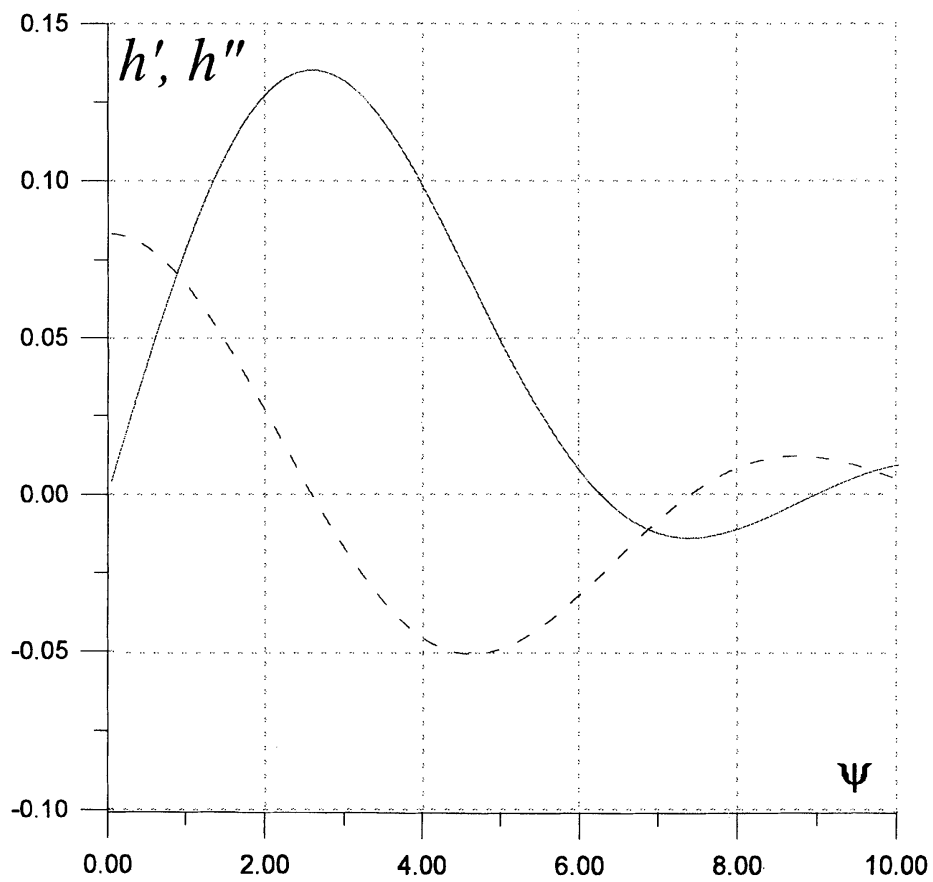

FIGURE 2 Phase factors $h^{\prime}(\psi)=\mathrm{d} h / \mathrm{d} \psi, h^{\prime \prime}=\mathrm{d}^{2} h / \mathrm{d} \psi^{2}$ with $h(\psi)=(\cos \psi-1) \psi^{-2}$ determine signs and values of longitudinal and transverse increment rates correspondingly.

$$
\left|\frac{Z_{\mathrm{sc}}^{\|}}{n}\right|=\frac{Z_{0} \ln \left(r_{\max } / a_{\mathrm{i}}\right)}{\gamma^{2} \beta}, \quad r_{\max }=\min b, k^{-1} .
$$

If the Keil-Schnell condition is not satisfied, the instability could be damped due to the cooling itself. This is possible for rather low ion currents, typical for a problem of beam crystallization. ${ }^{13,14}$ Both coherent increment and cooling decrement are proportional to the electron current. Therefore, the stability condition determines in this case the threshold ion current, but is independent of the electron one.

\section{TRANSVERSE IMPEDANCE}

According to a conventional concept, a back action of induced fields on a beam density perturbation can be described in terms of 


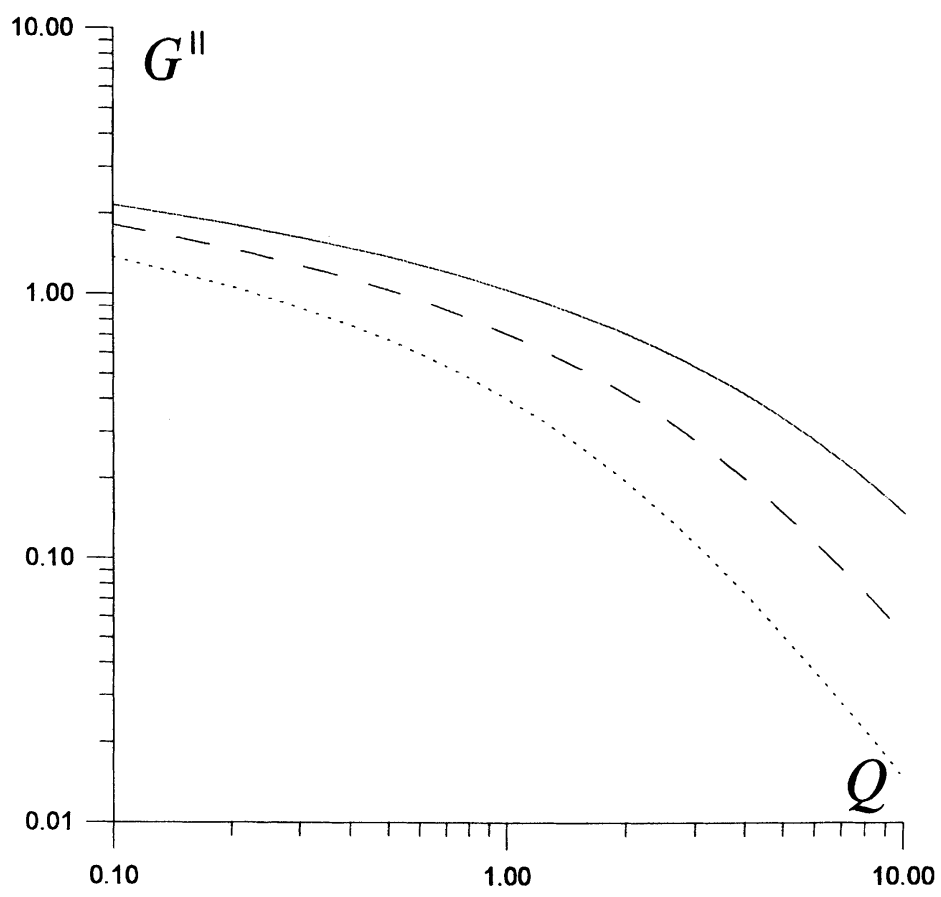

FIGURE 3 The formfactor $G^{\|}(Q)$ determines the value of the longitudinal increment. The sizes ratio $a_{\mathrm{i}} / a=0.1,0.2$ and 0.4 (solid, dash and dot lines).

impedances, for transverse degrees of freedom as well as for the longitudinal. For a dipole mode, the transverse impedance $Z^{\perp}$ is introduced as a kick response function on an oscillating dipole moment:

$$
-\frac{\partial\langle\Phi\rangle}{\partial x} \tau=i e \gamma \bar{\rho}_{\mathrm{i}} x_{\mathrm{i}} Z^{\perp}
$$

where $\bar{\rho}_{\mathrm{i}}$ is a linear density of the ion beam in the reference frame, $x_{\mathrm{i}}$ is an amplitude of its deviation along the $x$-direction, the potential $\langle\Phi\rangle$ is given by Eq. (20) with $l=1$ :

$$
\begin{aligned}
\langle\Phi\rangle= & -i \frac{2 \pi e}{\omega_{\mathrm{e}}^{2}} \cos \phi \sum_{\mu} J_{1}\left(\kappa_{\mu} r\right) u_{\mu}(k) \tilde{n}_{\mathrm{i} \mu} \\
& \times\left[\frac{u_{\mu+} \omega_{\mu}(k)}{\omega_{\mu}(k)-\omega_{\mathrm{i}}} T\left(\Delta \psi_{\mu+}\right)+\frac{u_{\mu-} \omega_{\mu}(k)}{\omega_{\mu}(k)+\omega_{\mathrm{i}}} T\left(\Delta \psi_{\mu-}\right)\right] .
\end{aligned}
$$


For the dipole mode the ion density perturbation $\tilde{n}_{\mathrm{i}}(r, \phi)=$ $\bar{n}_{\mathrm{i}}\left(x-x_{\mathrm{i}}\right)-\bar{n}_{\mathrm{i}}(x)=-x_{\mathrm{i}} \cos \phi \mathrm{d} \bar{n}_{\mathrm{i}} / \mathrm{d} r=\sum_{\mu} \tilde{n}_{\mathrm{i} \mu} J_{l}\left(\kappa_{\mu} r\right) \cos \phi ; \quad$ making use of the orthogonality conditions (10), the density Bessel-amplitudes write

$$
\tilde{n}_{\mathrm{i} \mu}=\frac{2 \kappa_{\mu} x_{\mathrm{i}}}{a^{2} F_{\mu}^{2}} \int \bar{n}_{\mathrm{i}}(r) J_{0}\left(\kappa_{\mu} r\right) r \mathrm{~d} r=\frac{\kappa_{\mu} \bar{J}_{0 \mu}}{\pi a^{2} F_{\mu}^{2}} x_{\mathrm{i}} \bar{\rho}_{\mathrm{i}} .
$$

Then, the derivative $\partial / \partial x$ is taken and the radial averaging have to be done:

$$
\begin{aligned}
\frac{\partial n_{\mathrm{i}}}{\partial x} & =\tilde{n}_{\mathrm{i} \mu} \frac{\widehat{\partial}}{\partial x}\left(\frac{x}{r} J_{1}\left(\kappa_{\mu} r\right)\right)=\tilde{n}_{\mathrm{i} \mu} \frac{\kappa_{\mu}}{2} \bar{J}_{0 \mu} \\
& =\frac{\kappa_{\mu}^{2} \mathcal{K}_{\mu}}{2 \pi a^{2}} x_{\mathrm{i}} \bar{\rho}_{\mathrm{i}}, \quad \mathcal{K}_{\mu}=\frac{\bar{J}_{0 \mu}^{2}}{F_{\mu}^{2}} .
\end{aligned}
$$

Substituting (38), (36) in the impedance definition (35), the result reads

$$
\begin{aligned}
\operatorname{Re} Z^{\perp}(k)= & \frac{\tau}{\gamma a^{2} \omega_{\mathrm{e}}^{2}} \sum_{\mu} \mathcal{K}_{\mu} \kappa_{\mu}^{2} u_{\mu} \\
& \times\left[\frac{u_{\mu+} \omega_{\mu}(k)}{\omega_{\mu}(k)-\omega_{\mathrm{i}}} T\left(\Delta \psi_{\mu+}\right)+\frac{u_{\mu-} \omega_{\mu}(k)}{\omega_{\mu}(k)+\omega_{\mathrm{i}}} T\left(\Delta \psi_{\mu-}\right)\right] .
\end{aligned}
$$

Assuming terms $\propto \alpha$ to be small enough to be neglected, it gives

$$
\begin{aligned}
& \operatorname{Re} Z^{\perp}(k)= \frac{Z_{0}}{4 \pi \beta \gamma a} \frac{\ell}{\gamma a} \sum_{\mu} \frac{\mathcal{K}_{\mu} \kappa_{\mu}^{2} \psi_{\mu}}{\kappa_{\mu}^{2}+q^{2}} \\
& \times\left[\frac{1-\cos \left(\psi_{\mu}-\psi_{\mathrm{i}}\right)}{\left(\psi_{\mu}-\psi_{\mathrm{i}}\right)^{2}}-\frac{1-\cos \left(\psi_{\mu}+\psi_{\mathrm{i}}\right)}{\left(\psi_{\mu}+\psi_{\mathrm{i}}\right)^{2}}\right], \quad q=k / \gamma, \\
& \psi_{\mu}=\omega_{\mu} \tau, \quad \psi_{\mathrm{i}}=q \delta v \tau+\omega_{\mathrm{b}} \tau
\end{aligned}
$$

The most important here is the dependence of the impedance on the betatron phase; assuming $\psi_{\mathrm{b}}=\omega_{\mathrm{b}} \tau<1$ and extracting a first-order term (40) over this small parameter, the corresponding impedance component follows: 


$$
\begin{aligned}
\operatorname{Re} Z^{\perp}(k) & =\frac{Z_{0} \psi_{\mathrm{b}}}{2 \pi \beta \gamma a} \frac{\ell}{\gamma a} S_{2}^{\perp}(q), \\
S_{2}^{\perp}(q) & =\sum_{\mu} \frac{\mathcal{K}_{\mu} \kappa_{\mu}^{2} \psi_{\mu}}{\kappa_{\mu}^{2}+q^{2}} \frac{\mathrm{d}}{\mathrm{d} \psi_{\mu}} \frac{\cos \psi_{\mu}-1}{\psi_{\mu}^{2}}, \quad \psi_{\mathrm{i}}<1, \\
\operatorname{Re} Z^{\perp}(k) & =\frac{Z_{0} \psi_{\mathrm{b}}}{2 \pi \beta \gamma a} \frac{\ell}{\gamma a} \frac{\mathrm{d}^{2}}{\mathrm{~d} \bar{\psi}_{\mathrm{i}}^{2}}\left(\frac{\cos \bar{\psi}_{\mathrm{i}}-1}{\bar{\psi}_{\mathrm{i}}^{2}}\right) S_{3}^{\perp}(q), \\
S_{3}^{\perp}(q) & =\sum_{\mu} \frac{\mathcal{K}_{\mu} \kappa_{\mu}^{2} \psi_{\mu}^{2}}{\kappa_{\mu}^{2}+q^{2}}, \quad \bar{\psi}_{\mathrm{i}}=q \delta v \tau, \quad \psi_{\mu}<1 .
\end{aligned}
$$

The plots of the mode sums are presented on Figures 4 and 5 for a particular case $\psi_{\mathrm{e}}=3.5$ and variable sizes ratio $a_{\mathrm{i}} / a$. For $\psi_{\mu} \ll \pi$, the sums differ on a constant factor: $S_{2}^{\perp}=S_{3}^{\perp} / 12$.

In fact, the term $\operatorname{Re} Z^{\perp} \propto \psi_{\mathrm{b}}$ corresponds to a dissipative contribution in the force applied on the ion beam. According to the definition (35), this force is proportional to the ion transverse velocity, actually $\propto i \operatorname{Re} Z^{\perp} x_{\mathrm{i}} \propto i \omega_{\mathrm{b}} x_{\mathrm{i}} \propto \dot{x}_{\mathrm{i}}$. A sign of a derivative $\left(\partial / \partial \psi_{\mathrm{b}}\right)$ $\operatorname{Re} Z^{\perp}$ determines a sign of decrements introduced in synchrobetatron ion modes by the considered interaction. The sign is positive, when the phase advances $\psi_{\mathrm{e}}, \psi_{\mathrm{i}}$ are not too high: $\psi_{\mathrm{e}} \leq 2 \pi, \psi_{\mathrm{i}} \leq 2.6$.

The sum factor $S_{3}^{\perp}$ is independent on the plasma frequency; the dependence of the factor $S_{2}^{\perp}$ on the plasma phase advance $\psi_{\mathrm{e}}$ is shown on Figure 6. The impedance reaches the maximum at $q \simeq$ $2 \pi \kappa_{1} / \psi_{\mathrm{e}}$, which is almost the same for the various phase advances $\psi_{\mathrm{e}}$.

\section{TRANSVERSE INSTABILITIES}

Real part of the transverse impedance can give rise to a damping or instability. Assuming the interaction discussed to be weak enough, $\Lambda_{m}<\Omega_{\mathrm{s}}$, the synchrotron modes of the bunched ion beam are welldefined. The growth rate of the $m$ th mode calculated for an air-bag distribution reads ${ }^{12}$ 


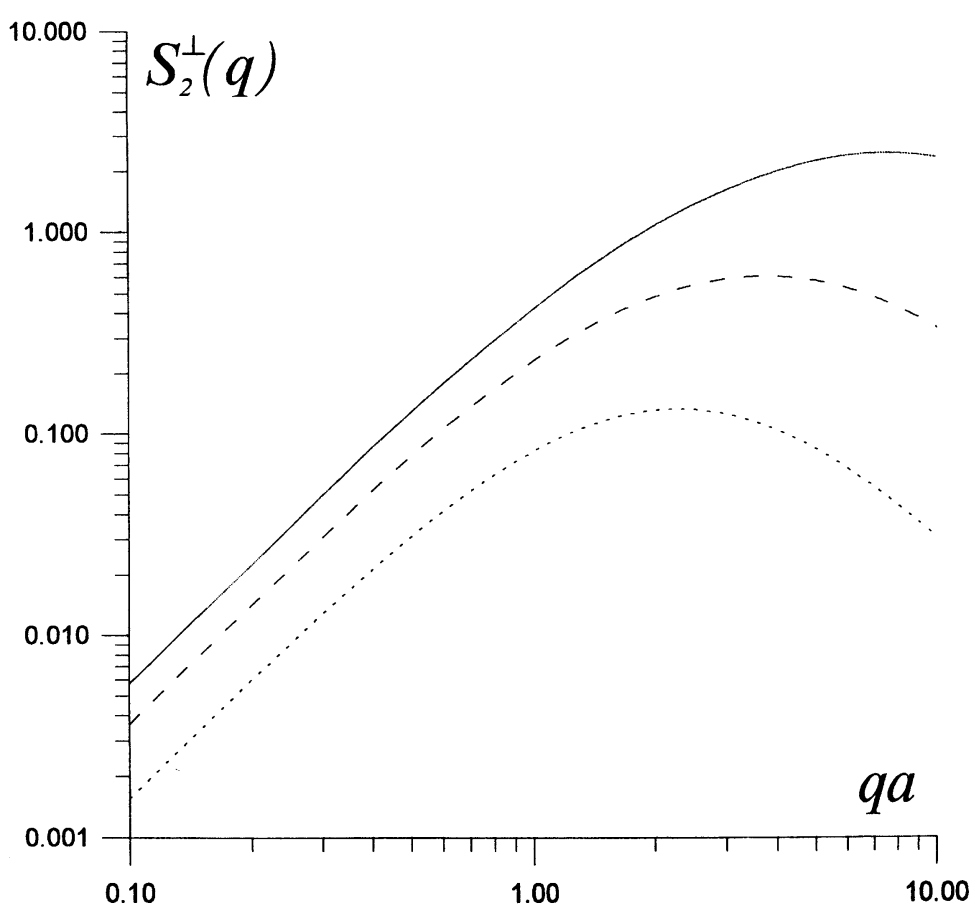

FIGURE 4 The transverse mode sum $S_{2}^{\perp}$ calculated for different beam sizes $a_{\mathrm{i}} /$ $a=0.1,0.2$ and 0.4 (the solid, dash and dot lines correspondingly). The Langmuir phase advance $\psi_{\mathrm{e}}=2.7$.

$$
\begin{aligned}
\Lambda_{m} & =-\frac{2 N r_{\mathrm{i}} c \beta_{x}}{\gamma \Pi} \int_{0}^{\infty} \mathrm{d} k \frac{\operatorname{Re} Z^{\perp}(k)}{Z_{0}} J_{m}^{2}\left(k l_{\mathrm{b}}\right) \\
& \approx-\frac{2 N r_{\mathrm{i}} c}{\pi \gamma l_{\mathrm{b}}} \frac{\ell}{\Pi \psi_{\mathrm{b}}} \int_{q_{m}}^{\infty} \frac{\mathrm{d} q}{q} \frac{\operatorname{Re} Z^{\perp}}{Z_{0}}, \quad q_{m}=\frac{m}{\gamma l_{\mathrm{b}}},
\end{aligned}
$$

where $\beta_{x}=\ell / \psi_{\mathrm{b}}$ is a beta-function in the location of the impedance. The real part of the impedance $(40) \operatorname{Re} Z^{\perp}(k) \propto \psi_{\mathrm{b}}=\omega_{\mathrm{b}} \tau$ means a dissipative force $F^{\perp} \propto i x Z^{\perp} \propto i \omega_{\mathrm{b}} x=-\dot{x}$ applied to the ion oscillations (35). If the integral (42) is positive, the collective ion motion decays due to the coherent interaction with electrons; otherwise an instability take place. The impedance (40) is positive when the phase advances $\psi_{\mathrm{e}} \leq 2 \pi, \psi_{\mathrm{i}} \leq 2.6$. However, even if $\psi_{\mathrm{e}}>2 \pi$, an integral 


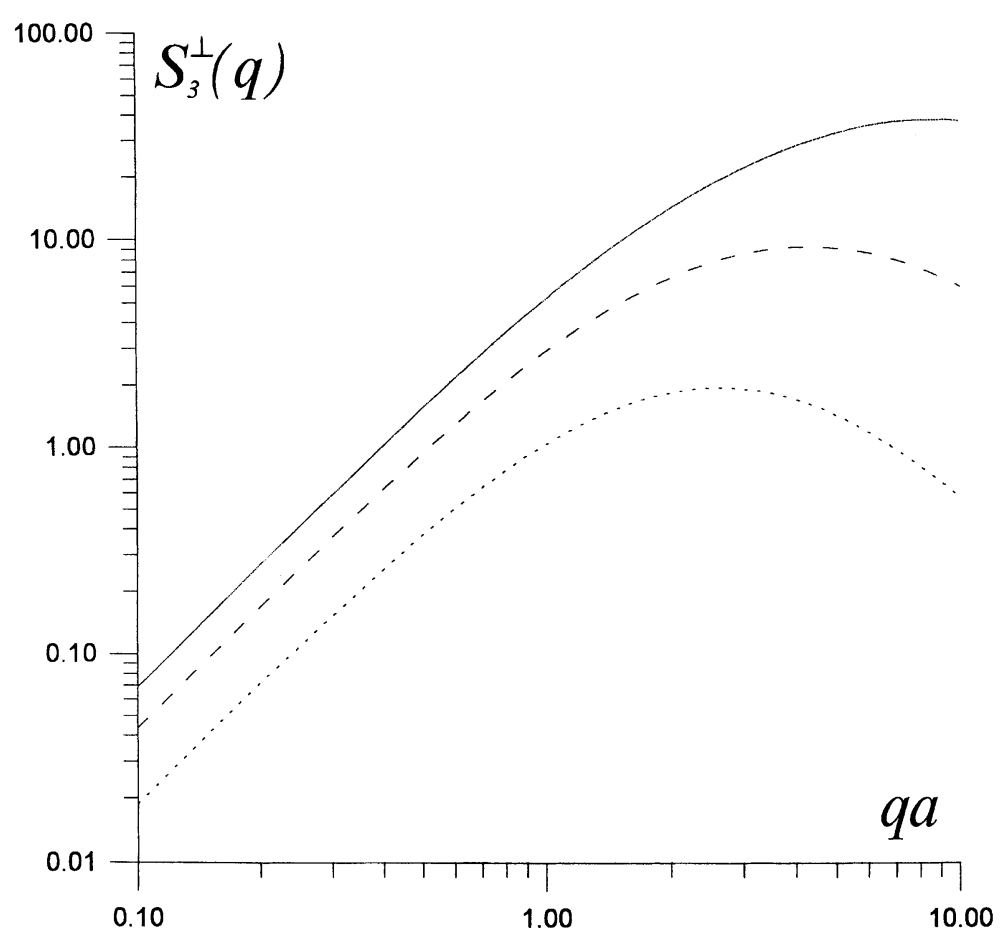

FIGURE 5 The mode sum $S_{3}^{\perp}$ at the same conditions as $S_{2}^{\perp}$ in a previous figure.

contribution of the intervals with the negative impedance cannot compete with the dominated positive contribution, which is demonstrated on Figure 6. Therefore, the plasma phase advance $\psi_{\mathrm{e}}$ does not restrict the stability area. Substituting in the integral (42) $Z^{\perp} \propto S_{3}^{\perp}$ (Eq. (41)), the transverse growth rate can be presented in the following way:

$$
\begin{gathered}
\Lambda_{m}=\frac{N r_{\mathrm{i}} c \psi_{\mathrm{e}}^{2}}{\pi^{2} \beta \gamma l_{\mathrm{b}} \Pi}\left(\frac{\ell}{\gamma a}\right)^{2} \tilde{\Lambda}, \quad \tilde{\Lambda}=-\int_{q_{m}}^{\infty} \frac{\mathrm{d} q}{q} h^{\prime \prime}\left(\psi_{\mathrm{i}}\right) S_{3}^{\perp}(q), \\
\psi_{\mathrm{i}}=q a \delta, \quad \delta=(\delta p / p)(\ell / \gamma a), \quad h^{\prime \prime}(\psi)=\frac{\mathrm{d}^{2}}{\mathrm{~d} \psi^{2}}\left(\frac{\cos \psi-1}{\psi^{2}}\right) .
\end{gathered}
$$

Up to this point, the detuning was not specified: is it a permanent velocity shift or oscillational one caused by the synchrotron motion. In the first case, the detuning phase advance $\psi_{\mathrm{i}} \propto q$ and the factor 


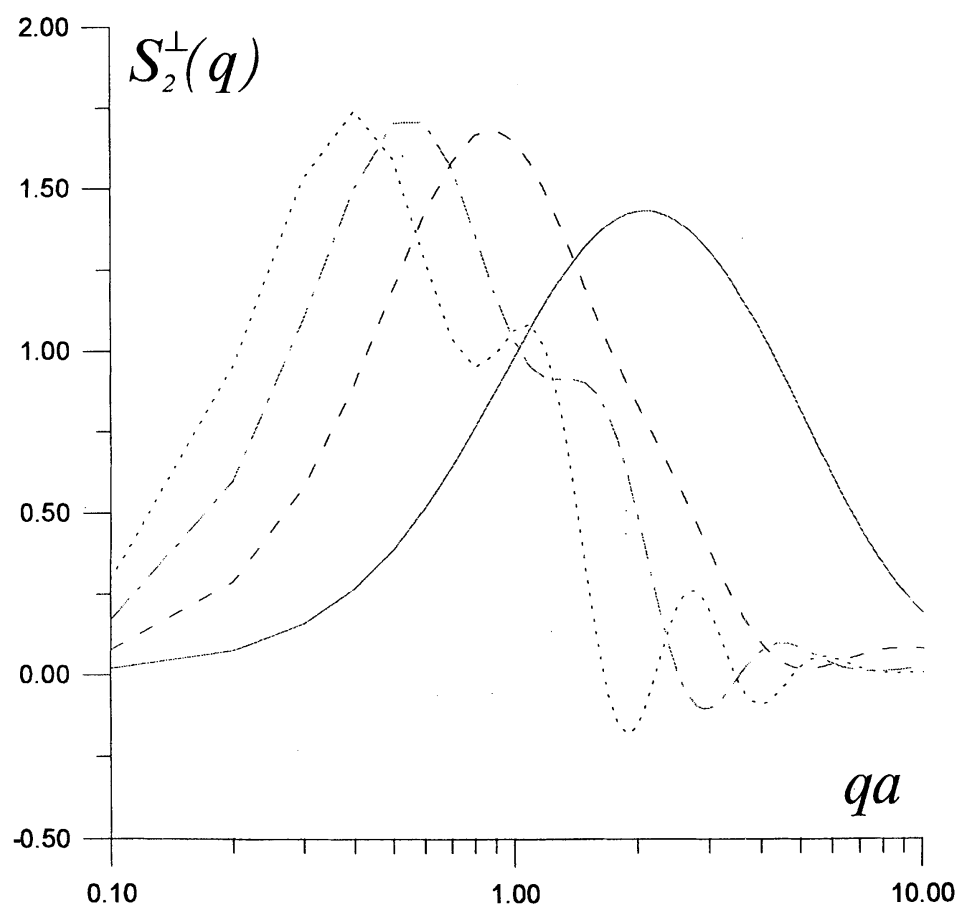

FIGURE 6 The mode sum $S_{2}^{\perp}$ for different plasma phase advances: $\psi_{\mathrm{e}} / 2 \pi=1,2,3$ and 4 (solid, dash, dash-dot and dot lines). The sizes ratio $a_{\mathrm{i}} / a=0.2$.

$h^{\prime \prime}\left(\psi_{\mathrm{i}}\right)$ has to be integrated in (43) over wave number $q$ together with the formfactor $S_{3}^{\perp}(q)$. In the second case the phase advance $\psi_{\mathrm{i}}$ has to be changed in (43) on a certain factor which does not depend on the wave number $q$, but is proportional to the mode number $m$ (31).

The increment rates (43) for the systematic detuning are presented in Figures 7 and 8 . The integral (42) with $Z^{\perp} \propto S_{3}^{\perp}$ converges at $q=k / \gamma \simeq 1 / a_{\mathrm{i}}$; the sufficient conditions of stability can be estimated as $\delta=(\delta p / p)(\ell / \gamma a) \leq 2 \cdot 2.6 a_{\mathrm{i}} / a \approx 5 a_{\mathrm{i}} / a$. According to the plots in Figures 7 and 8

$$
\delta_{\mathrm{th}}=\frac{\delta p_{\mathrm{th}}}{p} \frac{\ell}{\gamma a}=(5 \pm 1) a_{\mathrm{i}} / a \text { for } a_{\mathrm{i}} / a=0.1-0.4
$$

The increment (43) as a function of the detuning parameter $\delta$ reaches a maximum $\Lambda_{\max }$ at $\delta \simeq 2 \delta_{\text {th }}$. Taking into account that $S_{3}^{\perp} \sim\left(a / a_{\mathrm{i}}\right)^{2}$ 


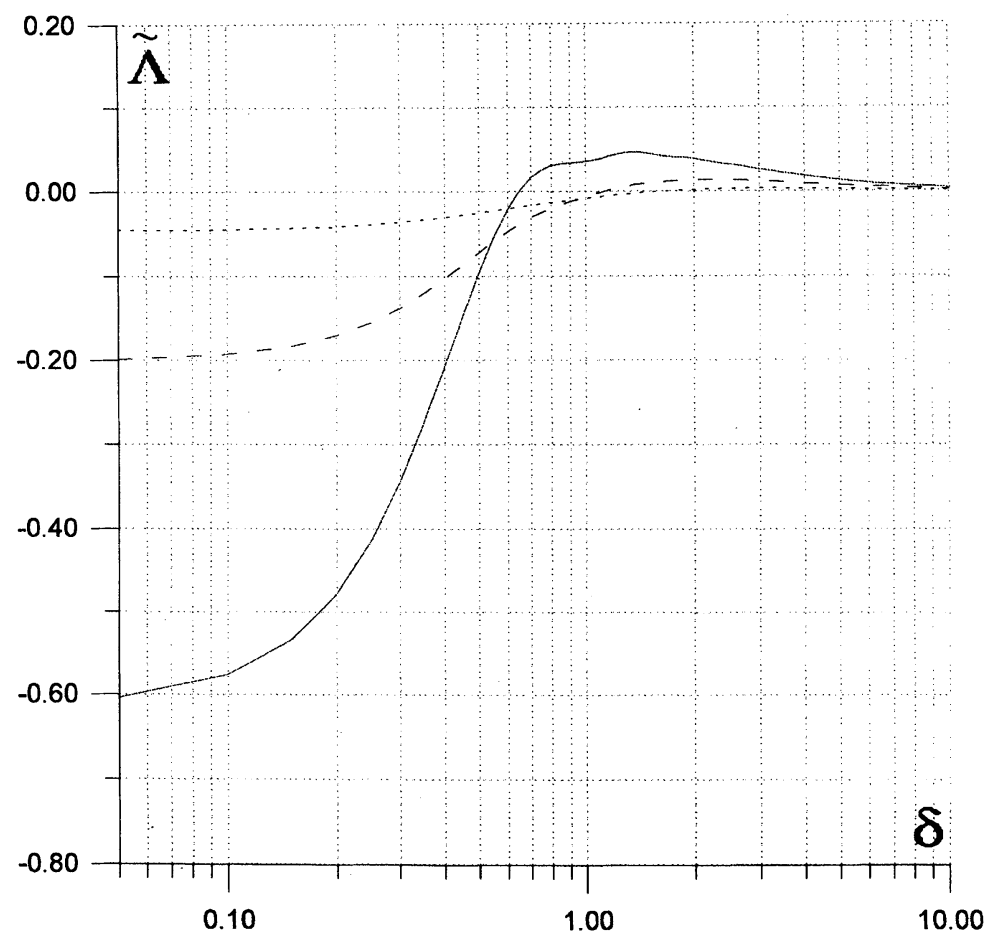

FIGURE 7 Factor $\tilde{\Lambda}$ specifies a growth rate of a transverse instability for a timeindependent velocity detuning. When the wind factor $\delta$ is small enough, all the synchrotron modes are damped; otherwise the blow-wind instability takes place. The solid, dash and dot lines correspond to $a_{\mathrm{i}} / a=0.1,0.2$ and 0.4 .

the maximum growth rate is found to be independent on the electron beam radius when $a / a_{\mathrm{i}} \gg 1, \Lambda_{\max } \sim a_{\mathrm{i}}{ }^{-2} \propto\left(\delta p_{\mathrm{th}} / p\right)^{-2}$.

The growth rate of this 'blow-wind' instability has been found in Ref. 8 for an infinite electron beam and flat ion beam; the result written in terms of (43) reads

$$
\tilde{\Lambda} \simeq 0.07 / \delta^{2}, \quad \delta v \gg \Delta_{\mathrm{e}}
$$

The growth rates (43) and (45) are compared in Figure 8. The latter is seen to cross the former ones close to their maxima, coinciding asymptotically with the case of equal r.m.s. dimensions, $a_{\mathrm{i}} / a=0.4$. According to (45), the motion is unstable when the detuning exceeds the width of electron distribution, which differs from the conclusion 


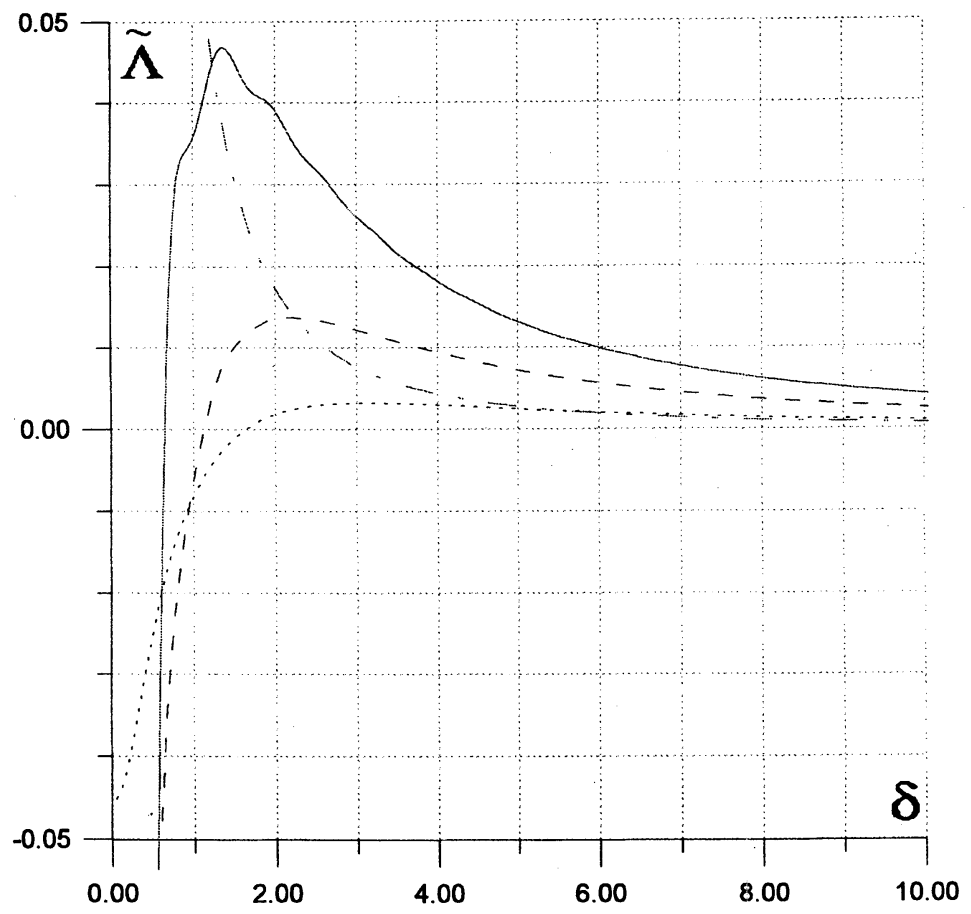

FIGURE 8 Solid, dash and dot lines are the same as in previous figure, a dash-dot line shows the result of Ref. 8.

(44) about stability at $\delta<\delta_{\text {th }}$. This threshold absence for zero-temperature electron beam in the result of Ref. 8 can be caused by the flat ion distribution assumed there. According to (44), $\delta_{\text {th }} \rightarrow 0$ when $a_{\mathrm{i}} \rightarrow 0$.

The increments (43) do not depend on the longitudinal mode number $m$ when $m<\gamma l_{\mathrm{b}} / a_{\mathrm{i}}$; thus, the instability taken place at $\delta \geq \delta_{\text {th }}$ cannot be damped due to the longitudinal frequencies dispersion. Also, it means a coherent damping below the threshold (44), with the same decrement for all the modes $m<\gamma l_{\mathrm{b}} / a_{\mathrm{i}}$, including $m=0$. This effect can be used for stabilizing single- and multi-bunch modes.

The 'blow-wind' instability could be an explanation for a particular phenomenon observed at CELSIUS. According to Ref. 4, a decrease of an ion lifetime with increase of electron current, or 'electron heating', observed at this ring looks to be caused by nonlinearity of an electric field of the electron beam beyond the beam 
radius, $r>a$. However, a fast dying out of the ion beam was also observed when it was deeply inside of the electron beam and detuned from it with $\delta p / p=0.02$. Substituting $\ell / a=200, \gamma=1, a_{\mathrm{i}} / a=0.2$ in Eq. (44), it gives the threshold $\delta p_{\text {th }} / p=0.005$, which means that the blow-wind instability have to take place for the detuning applied.

A comparison of the increments obtained (43) with the result of Dikansky and Pestrikov (45) is shown in Figure 8.

The increment (45) is seen to cross the lines (43) close to their maxima; asymptotically it looks to coincide with the growth rate for $a_{\mathrm{i}} / a=0.4$.

Turning to the case of the velocity detuning introduced by the synchrotron motion, the phase-dependent function has to be factor outside the integral sign (43), with $\psi_{\mathrm{i}}=2 \pi m / m_{\mathrm{i}}$, as in (31). The remaining integral can be taken, neglecting the weak dependence $\kappa(q)$ :

$$
\begin{gathered}
\Lambda_{m}=-\frac{N r_{\mathrm{i}} c \psi_{\mathrm{e}}^{2}}{2 \pi^{2} \beta \gamma l_{\mathrm{b}} \Pi}\left(\frac{\ell}{\gamma a}\right)^{2} h^{\prime \prime}(\psi) G^{\perp}(Q) ; \quad \psi=2 \pi \frac{m}{m_{\mathrm{i}}}, \quad Q=\frac{m}{m_{\mathrm{b}}}, \\
G^{\perp}(Q)=\sum_{\mu} \frac{\mathcal{K}_{\mu} \kappa_{\mu}^{2}}{\kappa_{\mu}^{2} a^{2}+Q^{2}} .
\end{gathered}
$$

Graphs of the functions $h^{\prime \prime}(\psi), G^{\perp}(Q)$ are presented on Figures 2 and 9.

The increment reaches a maximum at $\psi=\psi_{*} \approx 4.5, m \approx 0.7 m_{\mathrm{i}}$, where $h^{\prime \prime}=-h_{*}^{\prime \prime} \approx-0.05$ :

$$
\Lambda_{\max }=2.5 \cdot 10^{-3} \frac{N r_{\mathrm{i}} c \psi_{\mathrm{e}}^{2}}{\beta \gamma l_{\mathrm{b}} \Pi}\left(\frac{\ell}{\gamma a}\right)^{2} G^{\perp}\left(Q_{*}\right) ; \quad Q_{*} \approx 0.7 \frac{m_{\mathrm{i}}}{m_{\mathrm{b}}}=0.7 \frac{\gamma \eta a \Pi}{\nu_{\mathrm{s}} \ell l_{\mathrm{b}}} .
$$

Unlike the 'blow-wind' instability driven by the permanent detuning, this one is microwave, it can take place for synchrotron modes $m \simeq m_{\mathrm{i}}=\gamma^{2} \eta \Pi /\left(\nu_{\mathrm{s}} \ell\right)$ or higher.

Landau damping of this motion can be considered as a slow process if the dispersion of the synchrotron tunes $\Delta \nu_{\mathrm{s}}$ is small enough: $m_{\mathrm{i}} \Delta \nu_{\mathrm{s}} \ll \nu_{\mathrm{s}}$. Typically it can be satisfied only in the very close vicinity of the critical energy. The motion is not stabilized by the frequency dispersion if the last one is less than the Laslett tune shift, 


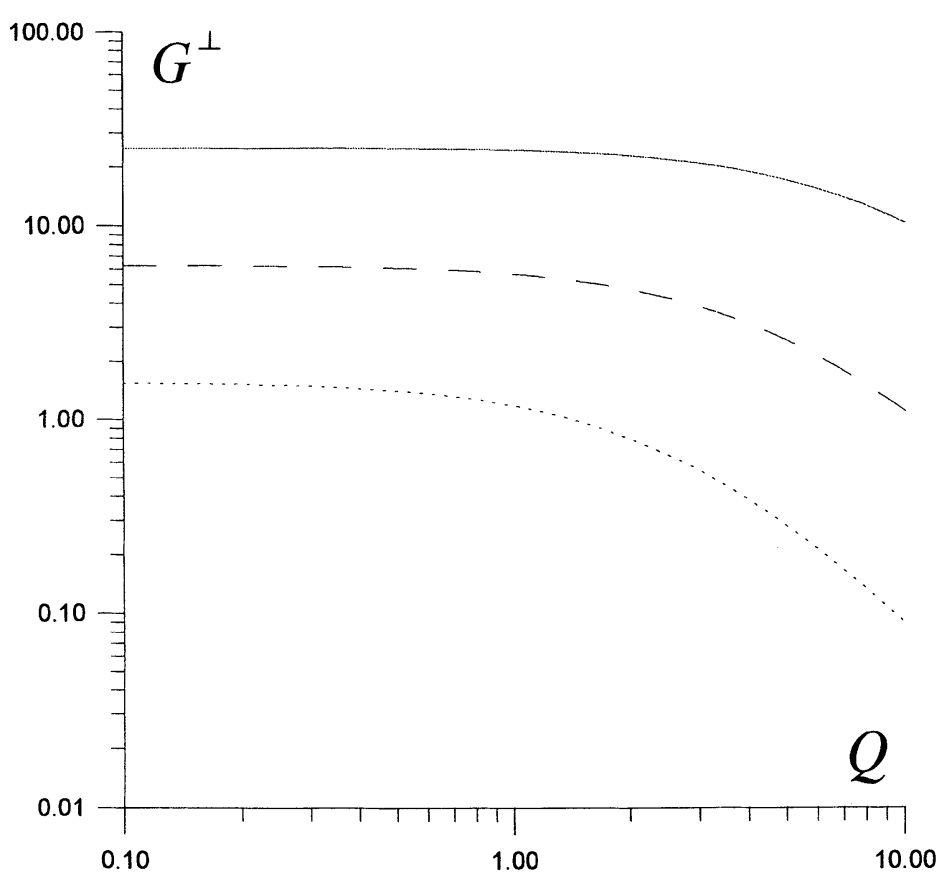

FIGURE 9 The transverse formfactor $G^{\perp}$ can be seen strongly dependent on the sizes ratio, $\propto\left(a / a_{\mathrm{i}}\right)^{2}$.

$m_{\mathrm{i}} \Delta \nu_{\mathrm{s}}<\Delta \nu_{\mathrm{L}}$. Therefore, in the close vicinity of the critical energy, where

$$
|\eta|<\frac{\nu_{\mathrm{s}}^{2} \ell}{\gamma^{2} \Pi \Delta \nu_{\mathrm{s}}}
$$

and

$$
|\eta|<\frac{\nu_{\mathrm{s}} \Delta \nu_{\mathrm{L}} \ell}{\gamma^{2} \Pi \Delta \nu_{\mathrm{s}}}
$$

the transverse oscillations of the ion bunch are always unstable.

In a case of a fast mixing of the synchrotron phases, when $m_{\mathrm{i}} \Delta \nu_{\mathrm{s}}>\nu_{\mathrm{s}}$, or

$$
|\eta|>\frac{\nu_{\mathrm{s}}^{2} \ell}{\gamma^{2} \Pi \Delta \nu_{\mathrm{s}}},
$$


the Landau damping can be considered in terms of a continuous beam. The motion is stabilized, when the dispersion of revolution frequencies compensate a particle-wave detuning due to the Laslett shift: $n \Delta \omega_{0}>\Delta \nu_{\mathrm{L}} \omega_{0}, n \simeq \bar{R} \gamma / a_{\mathrm{i}}$, which gives a condition of the stabilization due to the fast Landau damping:

$$
|\eta|>\frac{\Delta \nu_{\mathrm{L}} \ell}{\gamma^{2} \Pi} .
$$

Thus, the transverse oscillations are unstable, first, when the ionelectron velocity detuning is too high. A reason for this detuning could be the space charge of the ion beam, changing the electron momenta proportionally to a local ion current. ${ }^{16}$ This effect could be dangerous for future long relativistic coolers. Second, the instability takes place in the close vicinity of the critical energy. In all other cases, the electron cooler introduces the coherent damping in all the synchrobetatron modes, stabilizing single- and coupled-bunch motion.

\section{STRONG MICROWAVE INTERACTION}

Till this point, the coherent frequency shifts introduced by the impedances were supposed to be small in comparison with the synchrotron frequency. However, the increments calculated under this assumption can be compared or even higher than the synchrotron frequency. In a framework of the air-bag model, the frequencies $\Omega$ and synchrotron amplitudes $\mathcal{A}_{m}$ are found from the following set of equations, valid for broad-band impedances of arbitrary strength:

$$
\begin{aligned}
\left(\Omega-\omega_{\mathrm{b}}-m^{\prime} \Omega_{\mathrm{s}}\right) \mathcal{A}_{m^{\prime}}= & -i \frac{N r_{\mathrm{i}} c \beta_{x}}{\gamma \Pi} \sum_{m^{\prime \prime}} \mathcal{A}_{m^{\prime \prime}} i^{m^{\prime}-m^{\prime \prime}} \\
& \times \int_{-\infty}^{\infty} \mathrm{d} k \frac{Z^{\perp}(k)}{Z_{0}} J_{m^{\prime}}\left(k l_{\mathrm{b}}\right) J_{m^{\prime \prime}}\left(k l_{\mathrm{b}}\right)
\end{aligned}
$$

For low-order modes, $m^{\prime}, m^{\prime \prime} \ll k l_{\mathrm{b}}$, the Bessel functions can be replaced by their asymptotic values, and the set (48) reduces to a single dispersion equation: 


$$
\begin{gathered}
1=\Delta \Omega_{0} \sum_{m^{\prime}} \frac{1}{\Omega-\omega_{\mathrm{b}}-2 m^{\prime} \Omega_{\mathrm{s}}}, \quad \text { for even modes, } \\
1=\Delta \Omega_{0} \sum_{m^{\prime}} \frac{1}{\Omega-\omega_{\mathrm{b}}-\left(2 m^{\prime}+1\right) \Omega_{\mathrm{s}}}, \text { for odd modes; } \\
\Delta \Omega_{0}=-i \frac{N r_{\mathrm{i}} c \beta_{x}}{\gamma \Pi} \int_{-\infty}^{\infty} \mathrm{d} k \frac{Z^{\perp}(k)}{Z_{0}} J_{m}^{2}\left(k l_{\mathrm{b}}\right) .
\end{gathered}
$$

Taken into account that contributions of two neighbor terms dominate in the sum (49), the equation obtained can be solved:

$$
\begin{gathered}
\Omega-\omega_{\mathrm{b}}-m \Omega_{\mathrm{s}}=\Delta \Omega_{0}+\Omega_{\mathrm{s}}-\sqrt{\Delta \Omega_{0}^{2}+\Omega_{\mathrm{s}}^{2}} \\
\operatorname{Im} \Omega=\operatorname{Im} \Delta \Omega_{0} \begin{cases}1, & \text { if }\left|\Delta \Omega_{0}\right| \ll \Omega_{\mathrm{s}}, \\
0.5 \Omega_{\mathrm{s}}^{2} /\left|\Delta \Omega_{0}\right|^{2}, & \text { if }\left|\Delta \Omega_{0}\right| \gg \Omega_{\mathrm{s}} .\end{cases}
\end{gathered}
$$

Thus, an increase of the interaction strength $\left|\Delta \Omega_{0}\right|$ above the synchrotron frequency have a paradoxical result in the decrease of the coherent increment (decrement) rates. In a particular, the effect of the fast coherent damping works in a whole strength, when the synchrotron frequency is higher than the introduced decrement,

$$
\Omega_{\mathrm{s}}>\left|\Delta \Omega_{0}\right|
$$

Otherwise, the damping exists, but not so strong.

An important conclusion from the Eq. (51) is that the stability of the ion-electron motion is determined by the sign of $\operatorname{Im} \Delta \Omega_{0}$ (Eq. (50)), does not matter how high is it. Only the value, but not the sign of the increment (decrement) rate is renormalized in the case of strong interaction.

\section{CONCLUSIONS}

According to the analysis above, the coherent ion-electron interaction normally does not deteriorate ion beam parameters. On the contrary, this interaction introduces coherent decrements in low-order longitudinal and transverse modes of the cooled ion beam. 
The electron cooler introduces increments in high-order longitudinal beam modes. These microwave modes are stable when the Keil-Schnell condition with the ion space-charge impedance is satisfied, which is necessary with as well as without the electron cooler. Even more: the cooler itself stabilizes long-wave oscillations, remaining for the Landau damping only short waves, where the Coulomb logarithm of the space-charge impedance can be somewhat smaller.

A special case is a problem of ion cooling well below the KeilSchnell threshold, up to a liquid or crystalline state. If the KeilSchnell condition is not satisfied, the stability still could be trade off for the cooling itself. ${ }^{13,14}$ Both coherent increment and cooling decrement are proportional to the electron current. Therefore, the stability condition determines in this case the threshold ion current, but is independent on the electron one.

Transverse instabilities are entailed, when the detuning of the average ion velocity from the electron one is too high. Another reason for the transverse instability could be a vicinity to the critical energy. For both cases, the stability conditions do not include the electron current and are usually satisfied. Thus, the electron cooler introduces normally the coherent damping in the synchrobetatron motion.

This conclusion agrees with experimental findings of Refs. 4, 5, that the coherent ion-electron interaction cannot be responsible for the intensity phenomena observed at CELSIUS and IUCF. However, the coherent ion-electron instabilities could be dangerous for planned long relativistic coolers.

\section{Acknowledgements}

The author is thankful to N.S. Dikansky, V.V. Parkhomchuk, D.V. Pestrikov, D. Reistad, L. Tecchio, V. Vaccaro for fruitful discussions.

\section{References}

[1] G.I. Budker, Atomnaja Energiya, 22, 346 (1967).

[2] G.I. Budker et al., Part. Acc., 7, 197 (1976).

[3] I.N. Meshkov, Particles \& Nuclei, 25(6), 1487 (1994).

[4] D. Reistad et al., in Proc. of the Workshop on Beam Cooling and Related Topics, p. 327, Montreux, 1993. 
[5] T. Ellison et al., ibid., p. 377.

[6] T. Tanabe et al., ibid., p. 312.

[7] V.V. Parkhomchuk and D.V. Pestrikov, ibid., p. 327, Montreux, 1993.

[8] N.S. Dikansky and D.V. Pestrikov, The Physics of Intense Beams and Storage Rings, AIP Press, New York, 1994.

[9] A.V. Burov, in Proc. of the Workshop on Beam Cooling and Related Topics, p. 230, Montreux, 1993.

[10] A.V. Burov, in Proc. PAC-95, p. 3055, Dallas, 1995.

[11] A. Sessler and V. Vaccaro, CERN Report ISR-RF/67-2 (1967).

[12] A.W. Chao, Physics of Collective Beam Instabilities in High Energy Accelerators, J. Wiley \& Sons Inc., New York, 1993.

[13] A.V. Burov and V. Vaccaro, in Proc. of the Workshop on the Crystalline Beams, Erice, Italy, 1995.

[14] L. Tecchio et al., CRYSTAL. A Storage Ring for Beam Crystallization, Legnaro, Italy, 1995.

[15] N.S. Dikansky and D.V. Pestrikov, Preprint INP 76-40, Novosibirsk, 1976.

[16] N.S. Dikansky, private communication. 